\title{
Protective efficacy of inhaled quercetin for radiation pneumonitis
}

\author{
MENG QIN ${ }^{1}$, WEIJUAN CHEN ${ }^{2}$, JUANJUAN CUI $^{1}$, WENTAO LI $^{3}$, DONGMEI LIU $^{1}$ and WEIFEN ZHANG ${ }^{1}$ \\ ${ }^{1}$ Department of Pharmaceutics, College of Pharmacy, Weifang Medical University, Weifang, Shandong 261053; \\ ${ }^{2}$ Department of Pathology, People's Hospital of Shouguang, Weifang, Shandong 262700; ${ }^{3}$ Laboratory of Pharmacology, \\ College of Clinical Medicine, Weifang Medical University, Weifang, Shandong 261053, P.R. China
}

Received January 4, 2017; Accepted July 28, 2017

DOI: 10.3892/etm.2017.5290

\begin{abstract}
Radiation pneumonitis is a clinical problem with a high incidence. Once the onset of radiation pneumonitis has occurred, the administration of antioxidants and anti-inflammatory agents is the most commonly used method of clinical treatment. Quercetin (Que) is a common flavonoid, with potent anti-inflammatory and anti-oxidant activities. In the present study, the therapeutic effect of inhaled Que on radiation-induced radiation pneumonitis in rats was investigated. Treatment with Que via inhalation was shown to increase the number of leukocytes and erythrocytes in the blood, and reduce the number of inflammatory cells in bronchoalveolar lavage fluid. Histological examination of lung tissue indicated that inhaled Que reduced hemorrhaging and the infiltration of inflammatory cells, and suppressed the expression of the proinflammatory cytokines transforming growth factor- $\beta_{1}$ and interleukin-6. These results indicated that treatment with Que via inhalation ameliorates radiation pneumonitis by reducing the number of inflammatory cells, and attenuating the inflammatory response and pathological changes. This suggests that administration of Que via inhalation has the potential to become a novel treatment for radiation pneumonitis.
\end{abstract}

\section{Introduction}

Lung cancer is a major cause of cancer-related mortality worldwide (1), despite advances in surgery, radiotherapy and chemotherapy. The majority of patients present at an advanced stage of the disease, and advanced lung cancer remains an important area of research (2). Advanced lung cancer is frequently treated with radiation; however, the use of high radiation doses and large lung volumes results in a high likelihood of radiation pneumonitis, which is defined as inflammation in the lung following radiation $(3,4)$. Radiation

Correspondence to: Professor Weifen Zhang, Department of Pharmaceutics, College of Pharmacy, Weifang Medical University, 7166 Baotong West Street, Weifang, Shandong 261053, P.R. China E-mail: zhangwf@wfmc.edu.cn

Key words: quercetin, radiation pneumonitis, inhalation, rats, pharmacodynamics pneumonitis has become a clinical problem due to its high incidence, reported to be 29.6-50.3\% in Japan from 1983 to 1992 , and due to the difficulty in treating it $(5,6)$. Previous studies have demonstrated that antioxidant molecules are able to protect the lung from radiation-induced damage $(7,8)$. There has been a growing interest in the use of natural antioxidants derived from plants as additives in cosmetics and food (9), and traditional Chinese medicines, including quercetin (Que; 3,3,4,5,7-pentahydroxyflavone), curcumin and resveratrol are able to mitigate the inflammation induced by irradiation $(10,11)$.

Que is a member of the flavonol subclass of flavonoids, and is usually present in a glycosylated form (12). The major sources of Que are fruits, including apples, berries and cherries, vegetables, including onions and broccoli, and beverages, including red wine and tea (13). Que has been also identified in several medicinal plants, such as Ginkgo biloba, Aesculus hippocastanum and Hypericum perforatum (14). Que has been applied in various fields, due to its anti-inflammatory, antiviral, anti-allergy, antioxidant, anti-asthmatic and antitumor activities (15). Que has been reported to have protective effects against liver injury caused by chronic toluene exposure in rats (16). Despite this wide spectrum of pharmacological properties, the use of Que in the pharmaceutical field is limited due to its poor aqueous solubility, instability in physiological media and rapid photodegradation (17-19). The clinical application of Que is limited by its poor bioavailability and low stability in aqueous medium (20). The achievement of effective concentrations of Que in the lung tissue is difficult; therefore, higher doses and administration frequency are required, which leads to higher treatment costs as well as increased side effects, such as arthralgia and inflammatory response (21). Thus, it is necessary to develop new dosage forms and administration routes for Que with increased solubility and improved bioavailability. Inhalation therapy holds great potential for the effective treatment of various diseases, such as lung cancer. An inhalation therapy approach to respiratory disease, such as asthma, chronic obstructive pulmonary disease and lung cancer, is a promising means of improving therapeutic efficiency and minimizing unwanted systemic toxicity (22). A number of drugs, including dichromate flavonoid derivative and inhalative beclomethasone, have been investigated in vitro, in animal models and in human trials as targeted aerosol chemotherapies for lung cancer (23-25). Previous studies have also reported that lipid microparticles loaded with Que have 
enhanced stability in topical formulations (26-29). However, studies on the use of Que inhalation to treat radiation pneumonitis are lacking.

The aim of the present was to evaluate the preventive effect of inhaled Que on radiation pneumonitis using a rat model of induced radiation pneumonitis, and to identify a novel route of administration for Que.

\section{Materials and methods}

Materials. Pentobarbital sodium was purchased from Sinopharm Chemical Reagent Co., Ltd. (Shanghai, China); immunohistochemistry kits for the detection of interleukin (IL)-6 (cat no. EIA-1008) and transforming growth factor (TGF)- $\beta_{1}$ (cat no. EIA-1122) were supplied by Beijing Zhongshan Golden Bridge Biotechnology Co., Ltd. (Beijing, China); Que was supplied by Wuhan Tianyu Technology Co., Ltd. (Wuhan, China). Sodium dihydrogen phosphate and sodium hydrogen phosphate were purchased from Shanghai Chemical Reagent Co., Ltd. (Shanghai, China; batch nos. 20070604 and 20070509, respectively). All other chemicals and solvents were reagent grade.

Animals. A total of 48 male Wistar rats (5-6 weeks old; 150-180 g) were obtained from Shandong Lukang Animal Center (animal license no., Slxklu no. 2011002; Jining, China). The animals were maintained in a room at $23 \pm 2^{\circ} \mathrm{C}$, with a relative humidity of $50 \pm 5 \%$, and a 12 -h light/dark cycle. The rats were given a standard laboratory diet and water ad libitum. The study was approved by the Ethics Committee of Weifang Medical University (Weifang, China). All animal procedures were conducted in accordance with the rules and regulations approved by the Animal Care and Use Committee for Biological Studies of Shandong Province (Weifang, China).

Rat model of radiation pneumonitis. The $48 \mathrm{Wistar}$ rats were randomly divided into four groups (each $\mathrm{n}=12$ ) for treatment via inhalation as follows: i) No treatment (normal group); ii) $20 \mathrm{ml} 0.9 \%$ normal saline (model group); iii) $20 \mathrm{ml}$ inhaled Que at a dose of $10 \mathrm{mg} / 100 \mathrm{~g}$ (Que group), as previously described (30); and iv) $20 \mathrm{ml}$ dexamethasone at a dose of $5 \times 10^{-4} \mathrm{mg} / 100 \mathrm{~g}$ (positive control group). Rats in the normal, model, Que and positive control groups were exposed to X-ray irradiation of the pulmonary apex (15 Gy) using a 6-MV linear accelerator (ELEKTA AB, Stockholm, Sweden) to establish a radiation pneumonitis model. The $15-\mathrm{Gy}$ dose was delivered to the pulmonary apex in a single fraction. For administration via inhalation, animals were placed in a $15 \times 15 \times 20 \mathrm{~cm}$ Plexiglass box, and treatment was administered using an air compressor pump atomizer (PARI TurboBoy nebulizer; PARI GmbH, Starnberg, Germany) from a week before irradiation. Rats were administered the treatment by atomization for $30 \mathrm{~min}$ once a day, for 4 months.

Erythrocyte and leukocyte counts in blood. At 1, 2, 3 and 4 months, a quarter of the rats from each group were anesthetized with $0.3 \%$ pentobarbital $(3 \mathrm{mg} / 100 \mathrm{~g})$ by intraperitoneal injection, the chest was opened to expose the heart, and a $2 \mathrm{ml}$ sample of blood was taken from the heart. The blood was placed in an EDTA-K2 vacuum tube for measurement of the number of erythrocytes and leukocytes using a Sysmex KX-21 automated hematology analyzer (Sysmex Corporation, Osaka, Japan).

Bronchoalveolar lavage fluid (BALF) analysis. At 1,2,3 and 4 months, a quarter of the rats from each group were anesthetized and the trachea was cannulated. BALF was collected by flushing the lung with $2 \mathrm{ml}$ PBS via the trachea immediately following sacrifice. Approximately $5.0 \mathrm{ml}$ BALF was recovered following three lavages. The BALF was centrifuged $\left(4^{\circ} \mathrm{C}\right.$, $3,230 \mathrm{x} \mathrm{g}, 10 \mathrm{~min}$ ) and the supernatant was stored at $-80^{\circ} \mathrm{C}$. The pellet was resuspended in $5 \mathrm{ml}$ PBS. The number of leukocytes was measured using a Sysmex KX-21 automated hematology analyzer.

Histological analysis with hematoxylin and eosin staining. Following the collection of BALF, lung tissues were fixed in $10 \%$ paraformaldehyde for $24 \mathrm{~h}$ at room temperature, then paraffin-embedded, sectioned at 5-8 $\mu \mathrm{m}$ thickness, and subjected to routine hematoxylin (5 $\mathrm{min})$ and eosin staining (2-3 $\mathrm{min})(31)$ at room temperature for observation using a 37XC microscope (Shanghai Optical Instrument Factory Co., Ltd., Shanghai, China).

Immunohistochemistry. Immunohistochemical detection of IL- 6 and TGF- $\beta_{1}$ was performed using immunohistochemistry kits. The lung sections (prepared using the same steps mentioned above) were incubated with $0.3 \%$ hydrogen peroxide for $20 \mathrm{~min}$ at room temperature to block endogenous peroxidase activity, followed by rinsing with PBS, pH 7.4, and the absorption of excess fluid using filter paper. The sections were then treated with rabbit anti-murine IL-6 (EIA: 1008) or TGF- $\beta_{1}$ (EIA: 1122) antibody (1:10 dilution; Beijing Zhongshan Golden Bridge Biotechnology Co., Ltd., Beijing, China) overnight at $4^{\circ} \mathrm{C}$. Subsequently, the sections were treated with polymer enhancer (Beijing Zhongshan Golden Bridge Biotechnology Co., Ltd.) for $20 \mathrm{~min}$ at $37^{\circ} \mathrm{C}$, followed by rinsing with PBS and the absorption of excess fluid using filter paper. The sections were then treated with murine anti-rabbit antibody (1:100 dilution) for $30 \mathrm{~min}$ at $37^{\circ} \mathrm{C}$, washed and visualized by 3,3-diaminobenzidine tetrahydrochloride DAB. Finally, the sections were counter-stained with hematoxylin for $5 \mathrm{~min}$ at room temperature. For each section, five random microscopic fields were selected and observed. The total cell count and positive cell count were calculated in order to determine the percentage of positive cells. The positive cells were counted using the system: - -(strong negative sample), -(negative sample), + (positive sample) and ++ (strong positive sample).

Statistical analysis. Data are reported as the mean \pm standard deviation. Statistical analysis was performed using SPSS 13.0 software (SPSS, Inc., Chicago, IL, USA). Data were analyzed using one-way analysis of variance followed by the Least Significant Difference post hoc test. $\mathrm{P}<0.05$ was considered to indicate a statistically significant difference.

\section{Results}

Behavior and general observations. Rats in the normal group exhibited significant activity, fine and smooth fur, 
Table I. Effect of Que on radiation-induced erythrocytes in blood (mean \pm standard deviation, $\times 10^{12} / 1$ ).

\begin{tabular}{lcccc}
\hline $\begin{array}{l}\text { Time } \\
\text { (months) }\end{array}$ & Normal & Model & Que & $\begin{array}{c}\text { Positive } \\
\text { control }\end{array}$ \\
\hline 1 & $6.75 \pm 0.37$ & $7.38 \pm 1.15$ & $7.40 \pm 0.41$ & $6.89 \pm 0.34$ \\
2 & $7.23 \pm 0.16$ & $7.34 \pm 0.40$ & $7.04 \pm 0.10$ & $7.12 \pm 0.21$ \\
3 & $7.10 \pm 0.14$ & $6.88 \pm 0.29$ & $7.13 \pm 0.18$ & $7.43 \pm 0.11^{\mathrm{a}}$ \\
4 & $7.46 \pm 0.49$ & $7.91 \pm 0.89$ & $8.13 \pm 1.09$ & $7.63 \pm 0.51$ \\
\hline
\end{tabular}

${ }^{\text {a }} \mathrm{P}<0.05$ vs. the model group. Que, quercetin.

Table II. Effect of Que on radiation-induced leukocytes in blood (mean \pm standard deviation, $\mathrm{x} 10^{9} / 1$ ).

\begin{tabular}{lcccc}
\hline $\begin{array}{l}\text { Time } \\
\text { (months) }\end{array}$ & Normal & Model & Que & $\begin{array}{c}\text { Positive } \\
\text { control }\end{array}$ \\
\hline 1 & $7.87 \pm 1.20^{\mathrm{a}}$ & $4.84 \pm 0.74$ & $5.72 \pm 1.84$ & $4.62 \pm 0.84^{\mathrm{b}}$ \\
2 & $10.55 \pm 0.30^{\mathrm{a}}$ & $5.98 \pm 1.38$ & $6.80 \pm 0.95^{\mathrm{b}}$ & $6.82 \pm 0.92^{\mathrm{b}}$ \\
3 & $6.54 \pm 0.96^{\mathrm{a}}$ & $4.66 \pm 0.47$ & $5.08 \pm 0.72$ & $4.97 \pm 1.04$ \\
4 & $4.28 \pm 1.40$ & $4.93 \pm 0.58$ & $5.20 \pm 1.49$ & $4.68 \pm 0.24$
\end{tabular}

${ }^{\mathrm{a}} \mathrm{P}<0.05$ vs. the model group; ${ }^{\mathrm{b}} \mathrm{P}<0.05$ vs. the normal group. Que, quercetin.

and normal body weight. In all groups exposed to radiation, arched backs, piloerection, quietness and a reduction in body weight were observed in the first month. For rats in the Que group, the symptoms of arched back and piloerection were alleviated and body weight was gradually increased in the second month. Rats in the model group exhibited significantly delayed reactions, dispiritedness, and a reduction in body weight compared with the normal group. In the Que inhalation group, weight loss was prevented, and the rats had significantly higher body weights in comparison with the model group from the second month after irradiation until the end of the study. The positive control group was generally comparable with the Que group.

Erythrocyte and leukocyte counts in blood. Erythrocytes and leukocytes were counted following irradiation of the whole thorax (Tables I and II, respectively). There were a significantly higher number of erythrocytes in the positive control group compared with the model group at 3 months $(\mathrm{P}<0.05)$. However, the number of leukocytes was decreased following irradiation in the model, Que and positive control groups during the first three months. There was a significant difference in the number of leukocytes between the model and normal groups at 1,2 and 3 months $(\mathrm{P}<0.05)$, and no significant difference between the normal and Que groups at any time-point $(\mathrm{P}>0.05)$. Furthermore, at 4 months after irradiation, the numbers of erythrocytes and leukocytes in irradiated rats treated with Que was increased compared with those in the model group. These data indicate that Que inhalation ameliorates the inflammatory response in the blood of rats following whole-thorax irradiation.
Table III. Effect of Que on radiation-induced leukocytes in bronchoalveolar lavage fluid (mean \pm standard deviation, $\left.\mathrm{x} 10^{9} / 1\right)$.

\begin{tabular}{lcrcc}
\hline $\begin{array}{l}\text { Time } \\
\text { (months) }\end{array}$ & Normal & Model & Que & $\begin{array}{c}\text { Positive } \\
\text { control }\end{array}$ \\
\hline 1 & $2.82 \pm 0.95^{\mathrm{a}}$ & $5.80 \pm 0.89$ & $2.52 \pm 0.79^{\mathrm{a}}$ & $2.62 \pm 0.48^{\mathrm{a}}$ \\
2 & $2.47 \pm 1.06^{\mathrm{a}}$ & $10.55 \pm 1.47$ & $2.94 \pm 1.27^{\mathrm{a}}$ & $2.42 \pm 0.12^{\mathrm{a}}$ \\
3 & $2.44 \pm 0.05^{\mathrm{a}}$ & $6.98 \pm 1.04$ & $1.31 \pm 0.25^{\mathrm{a}}$ & $2.37 \pm 1.54^{\mathrm{a}}$ \\
4 & $2.58 \pm 0.22^{\mathrm{a}}$ & $4.81 \pm 0.47$ & $1.10 \pm 1.13^{\mathrm{a}, \mathrm{b}}$ & $2.78 \pm 0.54^{\mathrm{a}}$
\end{tabular}

${ }^{\mathrm{a}} \mathrm{P}<0.05$ vs. the model group; ${ }^{\mathrm{b}} \mathrm{P}<0.05$ vs. the normal group. Que, quercetin.
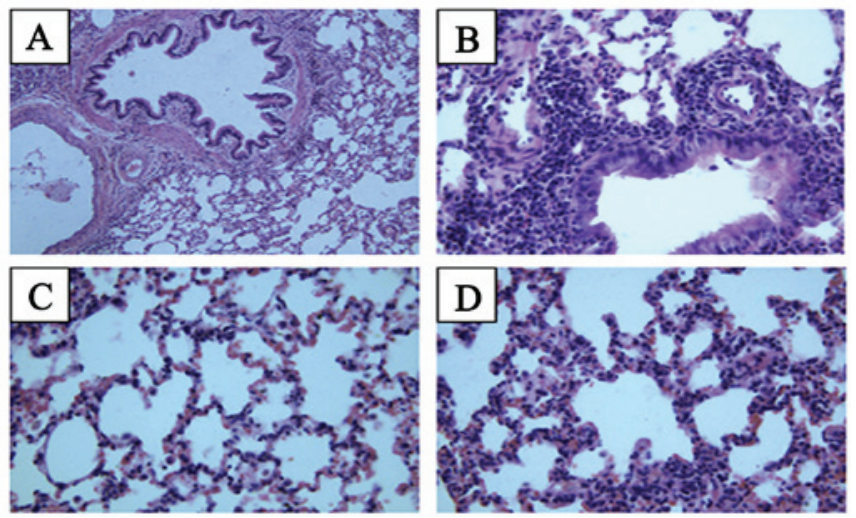

Figure 1. Pathological images of rat lung tissue at 2 months after irradiation. (A) Normal group, (B) model group, (C) positive control group and (D) quercetin group. Hematoxylin and eosin staining. Magnification, x400.

Leukocytes in BALF. To evaluate the effect of Que on inflammatory cells in BALF, leukocytes were measured following irradiation of the whole thorax (Table III). The number of leukocytes in the BALF peaked in the model group at 2 months after irradiation, and then decreased at 3 and 4 months. At 2 months, the number of leukocytes in the model group was significantly increased compared with that in the normal group $(\mathrm{P}<0.05)$. However, no significant difference was observed between the Que and normal groups $(\mathrm{P}>0.05)$, or between the Que and positive control groups $(\mathrm{P}>0.05)$ at this time-point. There were significant differences in the number of leukocytes between the model group and normal, Que, positive control group at 3 months $(\mathrm{P}<0.05)$. At 4 months after irradiation, the number of leukocytes of the model and Que groups was markedly decreased compared with that at the first month.

Lung histopathological changes. To examine the changes in lung tissue following thoracic irradiation combined with Que treatment, the extent of lung inflammation was assessed histologically at 2 months (Fig. 1) and 4 months (Fig. 2) following irradiation. In the normal group, the lung tissue exhibited normal lung alveoli, normal alveolar septum and well-distributed alveolar walls at 2 months; infiltration of inflammatory cells was not evident, and slight hemorrhaging was observed. In the model group, infiltration of inflammatory cells was 
Table IV. Effect of Que on radiation-induced levels of TGF- $\beta_{1}$ and IL-6 in bronchoalveolar lavage fluid.

\begin{tabular}{lcccc}
\hline Cytokine & Normal & Model & Que & $\begin{array}{r}\text { Positive } \\
\text { control }\end{array}$ \\
\hline TGF- $\beta_{1}$ & -- & ++ & + & + \\
IL-6 & -- & ++ & + & + \\
\hline
\end{tabular}

Que, quercetin; TGF, transforming growth factor; IL, interleukin.
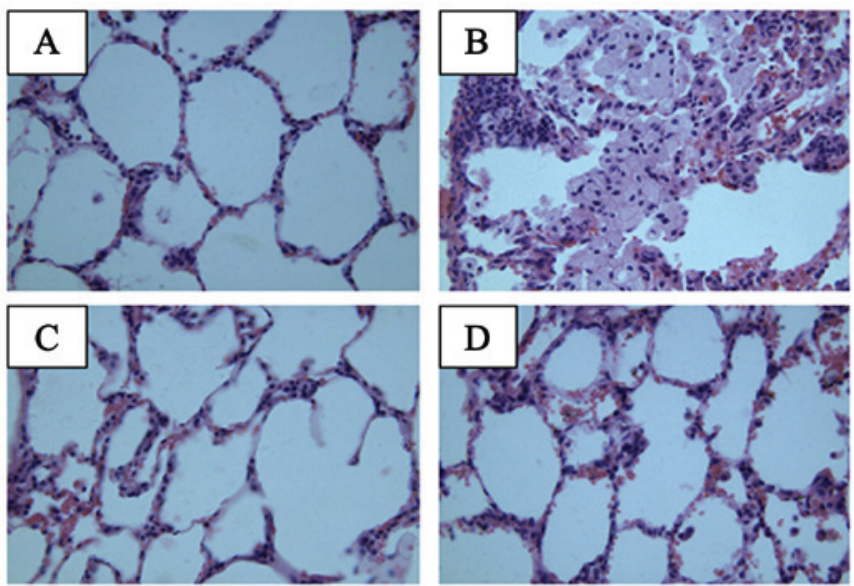

Figure 2. Pathological images of rat lung tissue at 4 months after irradiation. (A) Normal group, (B) model group, (C) positive control group and (D) quercetin group. Hematoxylin and eosin staining. Magnification, x400.

evident at 2 months after thoracic irradiation, and extensive hemorrhaging were observed. In the model group, lung injury was evident with destruction of the alveoli, thickened alveolar septum and decreased alveolar spaces. In the Que group, slight hemorrhages and normal lung alveoli were observed, and infiltration of inflammatory cells was not evident. There was no clear difference between the Que group and the positive control group. At 4 months, the lung tissue exhibited normal lung alveoli, thinning of the alveolar septum and increased alveolar spaces in the normal group. In the lung tissue of the model group, slight hemorrhages, thin alveolar septum and increased alveolar spacing were observed. However, Que clearly reduced the local inflammation caused by irradiation, and infiltration of inflammatory cells was not evident.

Levels of TGF- $\beta_{1}$ and IL- 6 . The levels of TGF- $\beta_{1}$ and IL-6 in the lung tissue were measured at 4 months after radiation (Figs. 3 and 4 and Table IV). In the normal group, brown staining indicating the presence of inflammatory cytokines was not visible. In the model group, the expression of TGF- $\beta_{1}$ was observed primarily in bronchial epithelial cells, peripheral lymphocyte and macrophages as brown staining of the lung tissue. However, the group treated with Que had only a small amount of brown staining of TGF- $\beta_{1}$ in the bronchial epithelial cells. Expression of IL- 6 was not observed in the normal group, as brown staining was not evident in the cytoplasm. In the model group, the expression of IL-6 was primarily observed in the alveolar space. The group treated with Que
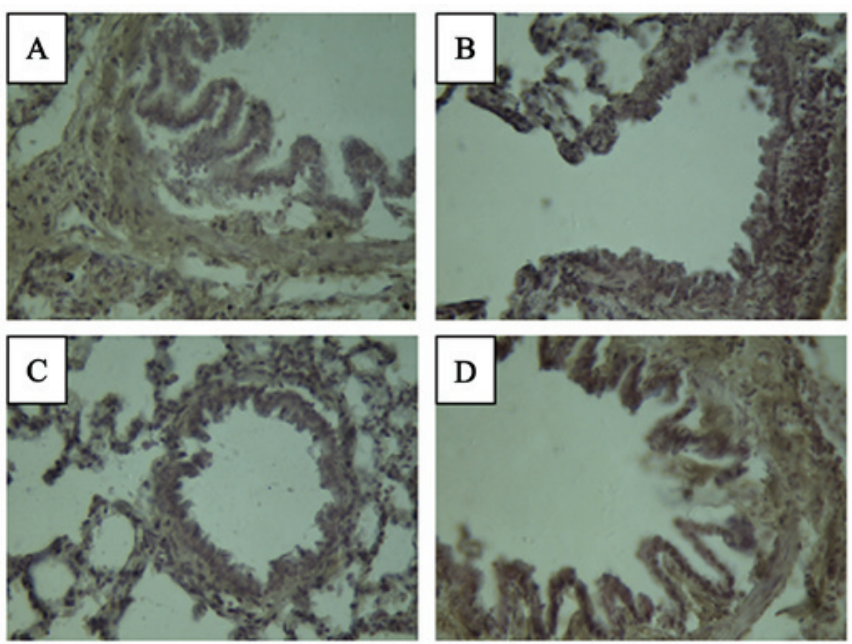

Figure 3. Transforming growth factor- $\beta_{1}$ immunohistochemical staining in lung tissue at 4 months after irradiation. (A) Normal group, (B) model group, (C) positive control group and (D) quercetin group. Magnification, $\mathrm{x} 400$.
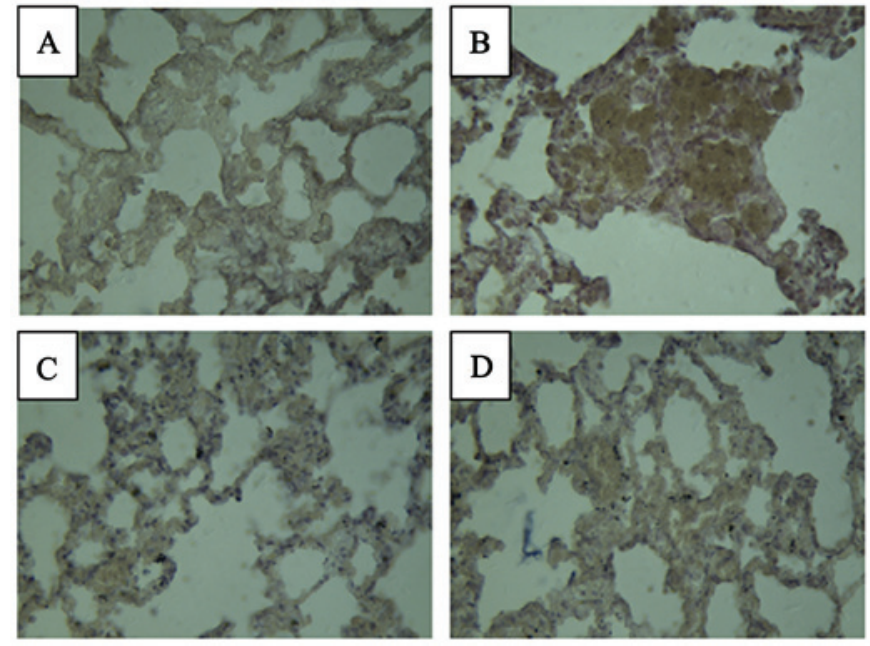

Figure 4. Interleukin-6 immunohistochemical staining in lung tissue at 4 months after irradiation. (A) Normal group, (B) model group, (C) positive control group and (D) quercetin group. Magnification, $\mathrm{x} 400$.

had only a small amount of brown staining of IL-6. No notable differences were observed in the levels of TGF- $\beta_{1}$ and IL-6 in the Que and positive control groups compared with the normal group.

\section{Discussion}

Radiation pneumonitis is a fairly common subacute side effect of radiotherapy in patients with lung cancer and the incidence is high. The incidence likely arises from different patient populations, the subjective scoring of radiation pneumonitis and treatment-related factors (32). In the present study, a rat model was used to investigate the effect of inhaled Que on the adverse effects of radiotherapy for lung cancer. The results demonstrated that the administration of Que by inhalation decreased inflammation in the lung. Previous studies have shown that rats survived pneumonitis following exposure to irradiation doses of 12,13 and 20 Gy $(33,34)$. In the present 
study, rats were exposed to radiation of the apex pulmonis (15 Gy) using 6-MV linearly accelerated X-rays to establish a radiation pneumonitis model. In the model group, numerous symptoms were evident following irradiation, including piloerection, arching of the back, quietness and weight loss, which demonstrated that the model was successful. Numerous studies have administered agents prior to radiation exposure to evaluate their ability to act as radiation protective agents, such as TNF- $\alpha$ and TGF- $\beta$ inhibitors $(35,36)$. However, it is necessary to investigate and identify agents that are effective for the reduction of radiation-induced lung injury when administered following irradiation (37).

The effects of Que on radiation-induced lung injury are unclear (30). The present study demonstrated that Que inhalation decreased the number of inflammatory cells in rats irradiated with X-rays. Que reduced the characteristics of inflammation, including the infiltration of inflammatory cells. The number of inflammatory cells, including lymphocytes (38), neutrophils (39) and alveolar macrophages (40) increases following irradiation. However, the effect of Que on airway inflammation remains largely unknown (30). Que has the ability to suppress radiation-induced inflammation and has beneficial effects on the lungs; however, it also has poor solubility, low bioavailability and low absorption $(41,42)$. In the present study, Que was administered by nebulization to maintain Que at high local concentrations (29). Drug delivery to the respiratory tract via inhalation is influenced by the properties of the inhaled particles as well as the breathing patterns of the experimental animals (43). The ability of a system to efficiently deliver a drug to the animal respiratory tract depends particularly on the inhaled particles' properties (44). Compressed air nebulizers produce aerosols with small-sized particles from drug-containing solutions and these aerosols have suitable characteristics for animal testing (27). In the present study, rats were treated using compressor pump atomization.

In all groups exposed to radiation, the rats exhibited arched backs, piloerection and a reduction in body weight. In the model group, infiltration of inflammatory cells was evident following thoracic irradiation, and extensive hemorrhaging was observed. Lung injury was manifested by destruction of the alveoli, thickened alveolar septa and decreased alveolar spaces. Histopathological examination revealed that Que reduced the radiation-induced histopathological changes compared with those in saline-treated model rats.

The present study demonstrated that irradiation of the thorax affected the chronic inflammatory response in the lung tissue. Previous studies have shown that pro-inflammatory cytokines, including TGF- $\beta_{1}$, IL- 6 and tumor necrosis factor- $\alpha$, are induced by irradiation of the thorax $(34,42)$. In the present study, the levels of TGF- $\beta_{1}$ and IL- 6 were elevated by lung tissue challenge with radiation, and were reduced in the Que group compared with the model group. Histopathological investigation revealed increased submucosal and airway wall thickening following irradiation. In the model group, the levels of TGF- $\beta_{1}$ and IL- 6 were increased compared with those in the normal group. The infiltration of inflammatory cells was markedly attenuated by Que administration. Brown staining of lymphocytes and macrophages was observed in the lung tissue of model group. However, the group treated with Que inhalation only had a small amount of brown staining. Radiation injury is characterized by capillary permeability, edema and excessive fibroblast proliferation within the alveolar septa $(34,42)$. The levels of TGF- $\beta_{1}$ and IL- 6 were markedly inhibited following Que administration compared with those in the model group. These results suggest that Que is an excellent candidate for use as an adjuvant therapy for radiation pneumonitis.

In conclusion, the present study demonstrated the preventive effect of Que inhalation on radiation pneumonitis in a rat model. The administration of Que using a nebulizer inhibited the inflammatory reaction of radiation pneumonitis by increasing the number of leukocytes and erythrocytes in the blood and decreasing the number of leukocytes in the BALF, and decreasing the levels of TGF- $\beta_{1}$ and IL- 6 . The results of histological observation also indicated that Que inhalation ameliorated radiation-induced hemorrhage, inflammation and fibrosis, and reduced inflammatory cell infiltration in the respiratory tract. These findings suggest that Que inhalation may be effective in protecting the lung tissue of radiation pneumonitis and is potentially clinically useful for use in patients with lung cancer who require radiotherapy.

\section{Acknowledgements}

The authors are grateful for generous financial support from the National Natural Science Foundation of China (grant nos. 81573717 and 81774125), the National Key Technology R\&D Program of the Ministry of Science and Technology (grant no. 2013GA740103), the Visiting Scholar Project of Weifang Medical University and project of Collaborative Innovation Center for Target Drug Delivery System of Weifang Medical University (no. 2017).

\section{References}

1. Parkin DM, Bray F, Ferlay J and Pisani P: Global cancer statistics, 2002.CA Cancer J Clin 55: 74-108, 2005.

2. Kuhar M, Sen S and Singh N: Role of mitochondria in quercetin-enhanced chemotherapeutic response in human non-small cell lung carcinoma H-520 cells. Anticancer Res 26: 1297-1303, 2006.

3. Graham MV, Purdy JA, Emami B, Harms W, Bosch W, Lockett MA and Perez CA: Clinical dose-volume histogram analysis for pneumonitis after 3D treatment for non-small cell lung cancer (NSCLC). Int J Radiat Oncol Biol Phys 45: 323-329, 1999.

4. Kwa SL, Lebesque JV, Theuws JC, Marks LB, Munley MT, Bentel G, Oetzel D, Spahn U, Graham MV, Drzymala RE, et al: Radiation pneumonitis as a function of mean lung dose: An analysis of pooled data of 540 patients. Int J Radiat Oncol Biol Phys 42: 1-9, 1998.

5. Nair MP, Mahajan S, Reynolds JL, Aalinkeel R, Nair H, Schwartz SA and Kandaswami C: The flavonoid quercetin inhibits proinflammatory cytokine (tumor necrosis factor alpha) gene expression in normal peripheral blood mononuclear cells via modulation of the NF-kappa beta system. Clin Vaccine Immunol 13: 319-328, 2006.

6. Watanabe H, Suga A, Tsuchihashi Y, Hori A, Kawakami K, Masaki H, Akiyama M, Ohishi K, Takahashi A, Nagatake T, et al: Clinical study of radiation pneumonitis over 10 years. Nihon Kyobu Shikkan Gakkai Zasshi 33: 384-388, 1995 (In Japanese).

7. Lee JC, Kinniry PA, Arguiri E, Serota M, Kanterakis S, Chatterjee S, Solomides CC, Javvadi P, Koumenis C, Cengel KA and Christofidou-Solomidou M: Dietary curcumin increases antioxidant defenses in lung, ameliorates radiation-induced pulmonary fibrosis, and improves survival in mice. Radiat Res 173: 590-601, 2010 
8. Wu L, Huang Z, Qin P, Yao Y, Meng X, Zou J, Zhu K and Ren G: Chemical characterization of a procyanidin-rich extract from sorghum bran and its effect on oxidative stress and tumor inhibition in vivo. J Agric Food Chem 59: 8609-8615, 2011.

9. Sharma SD, Meeran SM and Katiyar SK: Proanthocyanidins inhibit in vitro and in vivo growth of human non-small cell lung cancer cells by inhibiting the prostaglandin $\mathrm{E}(2)$ and prostaglandin $\mathrm{E}(2)$ receptors. Mol Cancer Ther 9: 569-580, 2010.

10. Comalada M, Camuesco D, Sierra S, Ballester I, Xaus J, Gálvez J and Zarzuelo A: In vivo quercitrin anti-inflammatory effect involves release of quercetin, which inhibits inflammation through down-regulation of the NF-kappaB pathway. Eur J Immunol 35: 584-592, 2005.

11. Bournival J, Plouffe M, Renaud J, Provencher C and Martinoli MG: Quercetin and sesamin protect dopaminergic cells from $\mathrm{MPP}^{+}$-induced neuroinflammation in a microglial (N9)-neuronal (PC12) coculture system. Oxid Med Cell Longev 2012: 921-941, 2012.

12. Grande F, Parisi OI, Mordocco RA, Rocca C, Puoci F, Scrivano L, Quintieri AM, Cantafio P, Ferla S, Brancale A, et al: Quercetin derivatives as novel antihypertensive agents: Synthesis and physiological characterization. Eur J Pharrm Sci 82: 161-170, 2016.

13. Boots AW, Haenen GR and Bast A: Health effects of quercetin: From antioxidant to nutraceutical. Eur J Pharmacol 585: 325-337, 2008.

14. Grande F, Parisi OI, Mordocco RA, Rocca C, Puoci F, Scrivano L, Quintieri AM, Cantafio P, Ferla S, Brancale A, et al: Quercetin derivatives as novel antihypertensive agents: Synthesis and physiological characterization. Eur J Pharm Sci 82: 161-170, 2016.

15. Brito AF, Ribeiro M, Abrantes AM, Pires AS, Teixo RJ Tralhão JG and Botelho MF: Quercetin in cancer treatment, alone or in combination with conventional therapeutics? Curr Med Chem 22: 3025-3039, 2015.

16. Kanter M: Protective effect of quercetin on liver damage induced by chronic toluene exposure in rats. Toxicol Ind Health 28: 483-491, 2012.

17. Fasolo D, Schwingel L, Holzschuh M, Bassani V and Teixeira H: Validation of an isocratic LC method for determination of quercetin and methylquercetin in topical nanoemulsions. J Pharm Biomed Anal 44: 1174-1177, 2007.

18. Kumari A, Yadav SK, Pakade YB, Singh B and Yadav SC: Development of biodegradable nanoparticles for delivery of quercetin. Colloids Surf B Biointerfaces 80: 184-192, 2010.

19. Aytac Z, Kusku SI, Durgun E and Uyar T: Quercetin/ $\beta$-cyclodextrin inclusion complex embedded nanofibres: Slow release and high solubility. Food Chem 197: 864-871, 2016.

20. Chimento A, Sala M, Gomez-Monterrey IM, Musella S, Bertamino A, Caruso A, Sinicropi MS, Sirianni R, Puoci F, Parisi OI, et al: Biological activity of 3-chloro-azetidin-2-one derivatives having interesting antiproliferative activity on human breast cancer cell lines. Bioorg Med Chem Lett 23: 6401-6405, 2013.

21. Zhao J, Liu J, Wei T, Ma X, Cheng Q, Huo S, Zhang C, Zhang Y, Duan $X$ and Liang XJ: Quercetin-loaded nanomicelles to circumvent human castration-resistant prostate cancer in vitro and in vivo. Nanoscale 8: 5126-5138, 2016.

22. Crompton G: A brief history of inhaled asthma therapy over the last fifty years. Prim Care Respir J 15: 326-331, 2006.

23. Otterson GA, Villalona-Calero MA, Sharma S, Kris MG, Imondi A, Gerber M, White DA, Ratain MJ, Schiller JH, Sandler A, et al: Phase I study of inhaled Doxorubicin for patients with metastatic tumors to the lungs. Clin Cancer Res 13: 1246-1252, 2007.

24. Otterson GA, Villalona-Calero MA, Hicks W, Pan X, Ellerton JA, Gettinger SN and Murren JR: Phase I/II study of inhaled doxorubicin combined with platinum-based therapy for advanced non-small cell lung cancer. Clin Cancer Res 16: 2466-2473, 2010

25. Moon H, Choi HH, Lee JY, Moon HJ, Sim SS and Kim CJ: Quercetin inhalation inhibits the asthmatic responses by exposure to aerosolized-ovalbumin in conscious guinea-pigs. Arch Pharm Res 31: 771-778, 2008.
26. Scalia S, Haghi M, Losi V, Trotta V, Young PM and Traini D: Quercetin solid lipid microparticles: A flavonoid for inhalation lung delivery. Eur J Pharm Sci 49: 278-285, 2013.

27. Scalia S, Trotta V, Traini D, Young PM, Sticozzi C, Cervellati F and Valacchi G: Incorporation of quercetin in respirable lipid microparticles: Effect on stability and cellular uptake on A549 pulmonary alveolar epithelial cells. Colloids Surf B Biointerfaces 112: 322-329, 2013.

28. Scalia S and Mezzena M: Incorporation of quercetin in lipid microparticles: Effect on photo- and chemical-stability. J Pharm Biomed Anal 49: 90-94, 2009.

29. Takashima K, Matsushima M, Hashimoto K, Nose H, Sato M, Hashimoto N, Hasegawa Y and Kawabe T: Protective effects of intratracheally administered quercetin on lipopolysaccharide-induced acute lung injury. Respir Res 15: 150, 2014.

30. Chang YC, Tsai MH, Sheu WH, Hsieh SC and Chiang AN: The therapeutic potential and mechanisms of action of quercetin in relation to lipopolysaccharide-induced sepsis in vitro and in vivo. PLoS One 8: e80744, 2013

31. Fischer AH, Jacobson KA, Rose J and Zeller R: Hematoxylin and eosin staining of tissue and cell sections. CSH Protoc 2008: pdb. prot4986, 2008.

32. Henkenberens C, Janssen S, Lavae-Mokhtari M,Leni K, Meyer A, Christiansen $\mathrm{H}$, Bremer $\mathrm{M}$ and Dickgreber N: Inhalative steroids as an individual treatment in symptomatic lung cancer patients with radiation pneumonitis grade II after radiotherapy - a single-centre experience. Radiat Oncol 11: 12, 2016.

33. Chiang CS, Liu WC, Jung SM, Chen FH, Wu CR, McBride WH, Lee CC and Hong JH: Compartmental responses after thoracic irradiation of mice: Strain differences. Int J Radiat Oncol Biol Phys 62: 862-871, 2005.

34. Rübe CE, Wilfert F, Palm J, König J, Burdak-Rothkamm S, Liu L, Schuck A, Willich N and Rübe C: Irradiation induces a biphasic expression of pro-inflammatory cytokines in the lung. Strahlenther Onkol 180: 442-448, 2004

35. Tsoutsou PG: The interplay between radiation and the immune system in the field of post-radical pneumonitis and fibrosis and why it is important to understand it. Expert Opin Pharmacother 15: 1781-1783, 2014.

36. Henkenberens C, Janssen S, Lavae-Mokhtari M, Leni K, Meyer A, Christiansen H, Bremer M and Dickgreber N: Inhalative steroids as an individual treatment in symptomatic lung cancer patients with radiation pneumonitis grade II after radiotherapy-a single-centre experience. Radiat Oncol 11: 12, 2016.

37. Pietrofesa R, Turowski J, Tyagi S, Dukes F, Arguiri E, Busch TM Gallagher-Colombo SM, Solomides CC, Cengel KA and Christofidou-Solomidou M: Radiation mitigating properties of the lignan component in flaxseed. BMC Cancer 13: 179, 2013.

38. Adawi A, Zhang Y, Baggs R, Rubin P, Williams J, Finkelstein J and Phipps RP: Blockade of CD40-CD40 ligand interactions protects against radiation-induced pulmonary inflammation and fibrosis. Clin Immunol Immunopathol 89: 222-230, 1998.

39. Thrall RS, Phan SH,McCormick JR and Ward PA: The development of bleomycin-induced pulmonary fibrosis in neutrophil-depleted and complement-depleted rats. Am J Pathol 105: 76-81, 1981.

40. Rubin P, Finkelstein J and Shapiro D: Molecular biology mechanisms in the radiation induction of pulmonary injury syndromes: Interrelationship between the alveolar macrophage and the septal fibroblast. Int J Radiat Oncol Biol Phys 24: 93-101, 1992.

41. Cai X, Fang Z, Dou J, Yu A and Zhai G: Bioavailability of quercetin: Problems and promises. Curr Med Chem 20: 2572-2582, 2013.

42. Liu H, Xue JX, Li X, Ao R and Lu Y: Quercetin liposomes protect against radiation-induced pulmonary injury in a murine model. Onocl Lett 6: 453-459, 2013.

43. Suarez S and Hickey AJ: Drug properties affecting aerosol behavior. Respir Care 45: 652-666, 2000.

44. Hrvacić B, Bosnjak B, Tudja M, Mesić M and Merćep M: Applicability of an ultrasonic nebulization system for the airways delivery of beclomethasone dipropionate in a murine model of asthma. Pharm Res 23: 1765-1775, 2006. 